\title{
Environmental Impacts of Using Energy Storage Aggregations to Provide Multiple Services
}

\author{
Abigail Kern \\ Department of Electrical Engineering \\ and Computer Science \\ University of Michigan \\ kerna@umich.edu
}

\author{
Jeremiah X. Johnson \\ Department of Civil, Construction, \\ and Environmental Engineering \\ North Carolina State University \\ jjohns24@ncsu.edu
}

\author{
Johanna L. Mathieu \\ Department of Electrical Engineering \\ and Computer Science \\ University of Michigan \\ jlmath@umich.edu
}

\begin{abstract}
Battery Energy Storage (BES) can provide multiple local and grid services. Previous work developed models and optimization approaches for allocating the power and energy capacities of multiple batteries to different tasks, enabling them to provide multiple services simultaneously. In this work, we explore the ability of batteries providing three local services to additionally participate in frequency regulation. We formulate a deterministic optimization problem to select optimal energy and power allocations for each BES to each service and then estimate the environmental impacts of aggregate battery multitasking. Specifically, we compare the environmental impacts (measured indirectly by the energy losses and required battery capacity) of multitasking batteries to single-service batteries providing the same set of services. Our results show that aggregated BES multitasking leads to significant reductions in total BES losses and BES capacity needed to perform the designated services. We also explore the sensitivity of the results to local/grid service prices.
\end{abstract}

\section{Introduction}

प

Many applications of battery energy storage (BES) have been proposed, including grid services such as frequency regulation [1] and generation resource adequacy [2], and local services such as industrial demand charge management [3] and backup power. It has been shown that performing multiple applications is preferable to improve battery utilization and net economic benefits [4, 5]. Although there are benefits to participating in grid services, individual BES systems may not be able to provide grid services for the

This research was funded by the UM Energy Institute and the National Science Foundation Environmental Sustainability Program Grant \#1510788. contracted duration due to the needs of their local service. In such cases, aggregation can help individual BES owners participate in grid services by sharing the committed capacity over more than one BES system, and over multiple time periods. Previous work has developed methods for co-optimizing the aggregated scheduling of multiple batteries to perform a local service and participate in the frequency regulation market [6, 7].

In this paper, we seek to understand the environmental impacts of using aggregations of distributed batteries to provide multiple local and grid services simultaneously as compared to using each battery to provide only a single service. We quantify environmental impacts indirectly by comparing the the energy losses due to charging/discharging and the reduction in required battery capacity to enable service provision required by multitasking batteries versus single-service batteries. We do this by formulating a deterministic optimization problem that schedules the energy/power capacities of multiple batteries to multiple services and then post-processing the results to assess the environmental impacts.

There are many examples of optimization of one battery for multiple services in existing literature. References [8] and [9] co-optimize the operation a single battery in the distribution system to perform multiple services using a mixed-integer linear program and linear program respectively. Uncertainty in day-ahead planning problems is incorporated through stochastic methods in [5] and [10]. Others have demonstrated through experiments the ability of a battery to perform different services simultaneously [11]. Aggregator services have also been investigated in conjunction with multitasking, such as using aggregated capacity from electric vehicles to provide frequency regulation in [6]. Reference [12] explored how combining peak shaving and frequency regulation increases revenue from BES operations, and showed 
that revenue from co-optimizing these two services was greater than the revenue of the individual services combined.

We also seek to investigate the benefits of using BES to perform multiple services. However, unlike [12], we do not focus on the economic benefit, but rather we investigate the potential for capacity reduction since larger capacities generally lead to larger life cycle environmental impacts. We also investigate the impact of aggregation/multitasking on BES losses. We acknowledge that uncertainty in weather forecasts cause uncertainty in both load consumption and solar generation profiles. Although an optimization method which considers uncertainty is important for BES day-to-day operation, in this paper, since we focus the environmental impacts of BES multitasking versus single-tasking rather than developing operational strategies, we use a deterministic formulation to simplify our analysis. Our future work will investigate stochastic optimization approaches and whether use of them changes the relative environmental impacts of multitasking vs. single-tasking.

The contributions of this paper are as follows. We extend the method developed in [7] to model three types of local services (mitigating photovoltaic (PV) clipping due to an undersized inverter, commercial or industrial $(\mathrm{C} / \mathrm{I})$ demand charge management, and residential customer time of use (TOU) price arbitrage) and optimize the energy/power capacity allocation to these local services and to one grid service: frequency regulation. We quantify the environmental impacts of multitasking by determining the reduction in installed BES capacity needed to perform identical levels of energy service and the reduction in system-wide roundtrip losses. We explore combinations of local services and the effect on aggregated bid and battery utilization, and how this affects the environmental impacts.

The remainder of this paper is organized as follows. In Section 2 we describe and formulate the optimization problem. Section 3 describes the three case studies performed and Section 4 describes their results. Section 5 concludes.

\section{Problem Description}

We wish to determine the amount of power and energy capacity allocated to each battery's local service and to frequency regulation (FR) in aggregate. The optimization is done from the point of view of an aggregator which is making both energy purchasing decisions as well as regulation market bid decisions on behalf of the individual participating BES systems. Each
BES system is characterized by the energy and power rating of its batteries, and a nominal load and/or PV generation profile.

In this section, we first list our notation and then describe three local services we model. Then we formulate the optimization problem and describe how we post-process the results to estimate losses.

\subsection{Notation}

\section{Indices}

$i \quad$ BES systems

$j$ Time steps of frequency regulation signal

$k$ Time steps within planning horizon

$l \quad$ Segments of linear approximations

$t$ Frequency regulation (FR) market interval

Sets

$\mathcal{N}$ Set of BES

$\mathcal{N}_{1} \quad$ Set of BES with Service 1

$\mathcal{N}_{2} \quad$ Set of BES with Service 2

$\mathcal{N}_{3} \quad$ Set of BES with Service 3

\section{System Parameters}

$\begin{array}{ll}a_{i, l}^{\mathrm{FR}, 1} & \text { Power coeff. used to compute } C_{i, k}^{\mathrm{FR}}[\$ / \mathrm{MW}] \\ a_{i, l}^{\mathrm{FR}, 2} & \text { Power coeff. used to compute } P_{i, k}^{\mathrm{eR}}[-] \\ b_{i, l}^{\mathrm{FR}, 1} & \text { Energy coeff. used to compute } C_{i, k}^{\mathrm{FR}} \\ b_{i, l}^{\mathrm{FR}, 2} & \text { [\$/MWh] } \\ E_{i}^{\text {cap }} & \text { Energy coeff. used to compute } P_{i, k}^{\mathrm{eFR}}[1 / \mathrm{h}] \\ G_{i, k} & \text { Energy capacity [MWh] } \\ g^{\mathrm{e}} & \text { PV peneration [MW] } \\ g_{k}^{\mathrm{e}, \mathrm{CI}} & \text { C/I TOU price [\$/MWh] } \\ g_{k}^{\mathrm{e}, \mathrm{es}} & \text { Residential TOU price [\$/MWh] } \\ g^{\mathrm{d}} & \text { C/I demand charge [\$/MW] } \\ K & \text { Number of time steps in planning horizon } \\ L_{i, k} & \text { Load [MW] } \\ M & \text { Large number } \\ P_{i}^{\mathrm{cap}} & \text { Power capacity [MW] } \\ p_{i}^{\mathrm{g}, \text { max }} & \text { Inverter limit [MW] } \\ r_{t}^{\mathrm{FR}} & \text { FR revenue [\$MW] } \\ S o C_{i}^{\mathrm{min}} & \text { Minimum state of charge [\%] } \\ S o C_{i}^{\mathrm{max}} & \text { Maximum state of charge [\%] } \\ S o C_{\mathrm{avg}}^{\mathrm{FR}} & \text { Average state of charge due to FR signal } \\ & \text { [\%] } \\ T & \text { Number of FR market intervals in planning } \\ & \text { horizon } \\ \Delta j & \text { FR signal time step duration } \\ \Delta k & \text { Planning horizon time step duration } \\ \eta_{i}^{\mathrm{c}} & \text { Charging efficiency [-] } \\ \eta_{i}^{\mathrm{d}} & \text { Discharging efficiency [-] }\end{array}$




\section{Decision Variables}

$\begin{array}{ll}B_{t}^{\mathrm{FR}} & \text { FR bid [MW] } \\ C_{i, k}^{\mathrm{FR}} & \text { Cost of providing FR [\$] } \\ C_{i}^{\mathrm{d}} & \text { Demand charge [\$] } \\ \Delta E_{i, k} & \begin{array}{l}\text { Energy transfer from local service to FR } \\ \text { [MWh] }\end{array} \\ E_{i, k}^{\mathrm{FR}} & \text { Energy allocation to FR [MWh] } \\ E_{i, k}^{\mathrm{l}} & \text { Energy allocation to local service [MWh] } \\ P_{i, k}^{\mathrm{FR}} & \text { Power allocation to FR [MW] } \\ P_{i, k}^{\mathrm{eFR}} & \text { Effective power allocation to FR [MW] } \\ P_{i, k}^{\mathrm{l}} & \text { Power allocation to local service [MW] } \\ p_{i, k}^{\mathrm{c}} & \text { Charging power for local service [MW] } \\ p_{i, k}^{\mathrm{d}} & \text { Discharging power for local service [MW] } \\ p_{i, k}^{\mathrm{g}} & \text { Power to the grid [MW] } \\ \bar{p}_{i}^{\mathrm{g}} & \text { Maximum demand [MW] } \\ p_{i, k}^{\mathrm{x}} & \text { PV curtailment [MW] } \\ y_{i, k} & \text { Binary decision to charge or discharge [-] }\end{array}$

Variables used for loss analysis

$\begin{array}{ll}E_{i, j}^{\text {bat }} & \text { Real-time BES energy state [MWh] } \\ E_{i}^{\text {loss }} & \text { Energy loss [MWh] } \\ p_{i, j}^{\text {bat }} & \text { Real-time BES power [MW] } \\ p_{i, j}^{\text {bat, }} & \text { Real-time BES charging power [MW] } \\ p_{i, j}^{\text {bat, }} & \text { Real-time BES discharging power [MW] } \\ \omega_{j}^{\text {FR }} & \text { Real-time FR signal [-] } \\ p_{i, j}^{\text {off }} & \text { Real-time offset power [MW] }\end{array}$

\subsection{Local Services}

Each battery performs one of three local services as shown in Fig. 1. Service 1 (Solar) stores solar energy that would otherwise have been clipped due to an undersized inverter. It discharges to the grid when the power limits of the inverter allow. This reflects the industry trend to undersize solar inverters [13] because the cost of the inverter is proportional to its power rating, and a solar array rarely generates its full nameplate rating [14]. However, with an undersized inverter, when the solar array is producing more than the inverter can convert, there is power lost through clipping. We assume the inverter is bi-directional and that there is no local load. Service $2(\mathbf{C} / \mathbf{I})$ is a commercial or industrial customer that uses BES to reduce its monthly demand charge. For this 24-hour optimization problem, we assume that the peak occurs on the day being examined. The industrial customer also has a TOU rate. Service 3 (Residential) is a residential customer that uses BES to arbitrage TOU prices. There is also a constraint applied which prevents the load from increasing more than twice the nominal load.

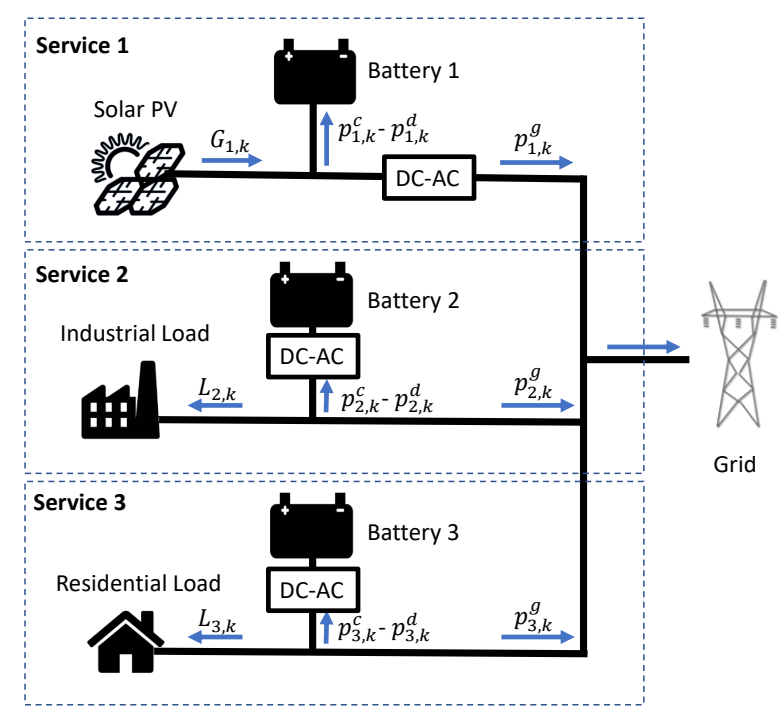

Figure 1. Three local services.

\subsection{Formulation}

We first formulate the optimization problem. The formulation is based on [7]. Key differences between the formulations are as follows: i) we consider different local services, ii) we do not allow load curtailment, iii) we do not model transformer overheating, iv) we constrain the final state of charge $(\mathrm{SoC})$ rather than valuing the energy in the battery at the end of the horizon, v) we ignore uncertainty, and vi) we enforce the complementarity constraint (i.e., no simultaneous charging and discharging), resulting in a mixed integer linear program.

The aggregator's objective is to maximize the total profit incurred from participation in the regulation market, while ensuring that each local service is performed. For every battery, the local service is related to minimizing costs of purchasing energy, or maximizing profit from selling energy to the grid. We formulate the following optimization problem for the aggregator:

$$
\begin{aligned}
\min _{\boldsymbol{x}} \sum_{t=1}^{T}-r_{t}^{\mathrm{FR}} B_{t}^{\mathrm{FR}} & +\sum_{k=1}^{K}\left\{\sum_{i \in \mathcal{N}} C_{i, k}^{\mathrm{FR}}-\sum_{i \in \mathcal{N}_{1}} g^{\mathrm{e}} p_{i, k}^{\mathrm{g}} \Delta k\right. \\
& +\sum_{i \in \mathcal{N}_{2}}\left\{g_{k}^{\mathrm{e}, \mathrm{CI}}\left(-p_{i, k}^{\mathrm{g}}\right) \Delta k+C_{i}^{\mathrm{d}}\right\} \\
& \left.+\sum_{i \in \mathcal{N}_{3}} g_{k}^{\mathrm{e}, \mathrm{res}}\left(-p_{i, k}^{\mathrm{g}}\right) \Delta k\right\}
\end{aligned}
$$

where the decision variable $\boldsymbol{x}$ includes the decision 
variables listed in the notation section over all associated indices $i, k, t$. The first term computes the (negative) revenue from frequency regulation. The second term computes the cost of providing frequency regulation, including the degradation effects and the cost of energy required to manage the battery state of charge, as explained in Section 2.3.2. The third term computes the (negative) revenue for batteries providing Service 1 (Solar). We assume the PV purchase price $g^{\mathrm{e}}$ is fixed over time. The fourth term computes the cost associated with Service $2(\mathrm{C} / \mathrm{I})$, specifically the cost of energy and the demand charge, where the latter is described in Section 2.3.4. The fifth term computes the cost associated with Service 3 (Residential), specifically the cost of energy.

2.3.1. Energy and Power Constraints. As in [7] we bound the energy state such that it remains within its physical limits

$$
\begin{aligned}
& S o C_{i}^{\mathrm{min}} E_{i}^{\mathrm{cap}} \leq E_{i, k}^{\mathrm{l}}+S o C_{i}^{\mathrm{min}} E_{i, k}^{\mathrm{FR}} \quad \forall i, k \\
& E_{i, k}^{\mathrm{l}}+S o C_{i}^{\max } E_{i, k}^{\mathrm{FR}} \leq S o C_{i}^{\max } E_{i}^{\mathrm{cap}} \quad \forall i, k
\end{aligned}
$$

where, like [7], we assume the energy state of the portion of the battery allocated to frequency regulation can vary between $S o C_{i}^{\mathrm{min}} E_{i, k}^{\mathrm{FR}}$ and $S o C_{i}^{\mathrm{max}} E_{i}^{\mathrm{FR}}$, where $E_{i, k}^{\mathrm{FR}}$ is the energy allocation to frequency regulation and $E_{i, k}^{1}$ is both the energy allocation to the local service and the energy state of the portion of the battery allocated to the local service. We also bound the energy state at the end of the hour

$$
\begin{aligned}
& S o C_{i}^{\mathrm{min}} E_{i}^{\mathrm{cap}} \leq E_{i, k}^{\mathrm{1}}+\left(\eta_{i}^{\mathrm{c}} p_{i, k}^{\mathrm{c}}-\frac{p_{i, k}^{\mathrm{d}}}{\eta_{i}^{\mathrm{d}}}\right) \\
&+S o C_{i}^{\mathrm{min}} E_{i, k}^{\mathrm{FR}} \quad \forall i, k \\
& E_{i, k}^{\mathrm{l}}+\left(\eta_{i}^{\mathrm{c}} p_{i, k}^{\mathrm{c}}-\frac{p_{i, k}^{\mathrm{d}}}{\eta_{i}^{\mathrm{d}}}\right)+S o C_{i}^{\mathrm{max}} E_{i, k}^{\mathrm{FR}} \\
& \leq S o C_{i}^{\max } E_{i}^{\mathrm{cap}} \quad \forall i, k
\end{aligned}
$$

and require the energy allocations to be positive

$$
\begin{array}{ll}
E_{i, k}^{1} \geq 0 & \forall i, k \\
E_{i, k}^{\mathrm{FR}} \geq 0 & \forall i, k .
\end{array}
$$

Similarly, we bound the power

$$
0 \leq P_{i, k}^{\mathrm{l}}+P_{i, k}^{\mathrm{FR}} \leq P_{i}^{\mathrm{cap}} \quad \forall i, k
$$

where $P_{i, k}^{\mathrm{l}}$ is the power allocated to the local service and $P_{i, k}^{\mathrm{FR}}$ is the power allocated to frequency control, and both must be positive

$$
\begin{array}{ll}
P_{i, k}^{\mathrm{FR}} \geq 0 & \forall i, k \\
P_{i, k}^{\mathrm{l}} \geq 0 & \forall i, k .
\end{array}
$$

We implement the complementarity constraint with

$$
\begin{aligned}
& p_{i, k}^{\mathrm{c}} \geq 0 \quad \forall i, k \\
& p_{i, k}^{\mathrm{d}} \geq 0 \quad \forall i, k \\
& p_{i, k}^{\mathrm{c}} \leq M y_{i, k} \quad \forall i, k \\
& p_{i, k}^{\mathrm{d}} \leq M\left(1-y_{i, k}\right) \quad \forall i, k \\
& y_{i, k} \in[0,1] \quad \forall i, k
\end{aligned}
$$

where $M$ is a number larger than the power rating of the battery. The power allocation to the local service is the greater of the charging and discharging power for the local service $P_{i, k}^{\mathrm{l}}=\max \left(p_{i, k}^{\mathrm{c}}, p_{i, k}^{\mathrm{d}}\right)$ which we implement as

$$
P_{i, k}^{\mathrm{l}} \geq p_{i, k}^{\mathrm{c}}+p_{i, k}^{\mathrm{d}} \quad \forall i, k .
$$

As in [7], we assume the energy state of the portion of the battery allocated to the local service evolves as

$$
E_{i, k+1}^{\mathrm{l}}=E_{i, k}^{\mathrm{l}}+\left(\eta_{i}^{\mathrm{c}} p_{i, k}^{\mathrm{c}}-\frac{p_{i, k}^{\mathrm{d}}}{\eta_{i}^{\mathrm{d}}}\right) \Delta k-\Delta E_{i, k+1} \quad \forall i, k
$$

where $\Delta E$ transfers energy from the portion of the battery allocated to the local service to the portion of the battery allocated to frequency regulation. Additionally, we assume that the energy state of the portion of the battery allocated to frequency regulation evolves as

$$
S o C_{\mathrm{avg}}^{\mathrm{FR}} E_{i, k+1}^{\mathrm{FR}}=S o C_{\mathrm{avg}}^{\mathrm{FR}} E_{i, k}^{\mathrm{FR}}+\Delta E_{i, k+1} \quad \forall i, k
$$

i.e., without energy transfers, the energy state would be the same at the end of each hour because state of charge variations due to frequency regulation are managed as described in Section 2.3.2. The energy transfer is bounded

$$
\begin{gathered}
\Delta E_{i, k} \leq E_{i, k}^{1} \quad \forall i, k \\
-\Delta E_{i, k} \leq S o C_{\mathrm{avg}}^{\mathrm{FR}} E_{i, k}^{\mathrm{FR}} \quad \forall i, k .
\end{gathered}
$$

We also assume that the battery is half full at the beginning and end of the planning horizon

$$
E_{i, k}^{\mathrm{l}}+S o C_{\mathrm{avg}}^{\mathrm{FR}} E_{i, k}^{\mathrm{FR}}=0.5 E_{i}^{\mathrm{cap}} \quad \forall i, k=0, K .
$$

The power to the grid, not including, frequency regulation actions, is

$$
p_{i, k}^{\mathrm{g}}=p_{i, k}^{\mathrm{d}}-p_{i, k}^{\mathrm{c}}+G_{i, k}-p_{i, k}^{\mathrm{x}}-L_{i, k} \quad \forall i, k
$$


where PV curtailment is bounded

$$
0 \leq p_{i, k}^{\mathrm{x}} \leq G_{i, k} \quad \forall i, k .
$$

For Service 1 the power through the inverter is limited

$$
\left|p_{i, k}^{\mathrm{g}}+P_{i, k}^{\mathrm{FR}}\right| \leq p_{i}^{\mathrm{g}, \max } \quad \forall i \in \mathcal{N}_{1}, k
$$

where $p_{i}^{\mathrm{g}, \max }$ is the power rating of the solar (shared with BES) inverter. For Service 3 we bound the absolute value of $p_{i, k}^{\mathrm{g}}$ to be less than twice the nominal load

$$
\left|p_{i, k}^{\mathrm{g}}\right| \leq 2 L_{i, k} \quad \forall i \in \mathcal{N}_{3}, k
$$

For Services 2 and 3, we additionally assume that there is no compensation for power fed back into the grid. For these services, we specify the net power to the grid to be negative (net consumption of power).

$$
p_{i, k}^{\mathrm{g}} \leq 0 \quad \forall i \in \mathcal{N}_{2}, \mathcal{N}_{3}, k
$$

2.3.2. Cost of Providing Frequency Regulation. We assume state of charge variations due to frequency regulation and battery losses are managed by purchasing offset power [15], i.e., at specified intervals energy is purchased from or sold to the grid to return the energy state of the portion of each battery allocated to frequency regulation to $S o C_{\mathrm{avg}}^{\mathrm{FR}}$. The cost of purchasing offset power and the cost of battery degradation from participation in frequency regulation were quantified in [15] and approximated in [7] with a set of linear constraints

$$
C_{i, k}^{\mathrm{FR}} \geq a_{i, l}^{\mathrm{FR}, 1} P_{i, k}^{\mathrm{FR}}+b_{i, l}^{\mathrm{FR}, 1} E_{i, k}^{\mathrm{FR}} \quad \forall l, i, k
$$

which lower bound the near-convex cost of providing frequency regulation. We use the coefficients used in [7], given in Table 1 .

2.3.3. Frequency Regulation Bid. Some of the power capacity of the battery must be saved to enable offsetting, which decreases the amount of capacity that can be used for frequency regulation. As in [7], we model the effective frequency regulation power capacity with a set of linear constraints

$$
P_{i, k}^{\mathrm{eFR}} \leq a_{i, l}^{\mathrm{FR}, 2} P_{i, k}^{\mathrm{FR}}+b_{i, l}^{\mathrm{FR}, 2} E_{i, k}^{\mathrm{FR}} \quad \forall l, i, k .
$$

Example constraints are shown in Fig. 2, Again, we use the coefficients used in [7], also given in Table 1.

When the frequency regulation market intervals $t$ are equivalent to the time steps $k$ then the frequency regulation bid is

$$
B_{t}^{\mathrm{FR}}=\sum_{i \in \mathcal{N}} P_{i, t}^{\mathrm{eFR}} \quad \forall t
$$

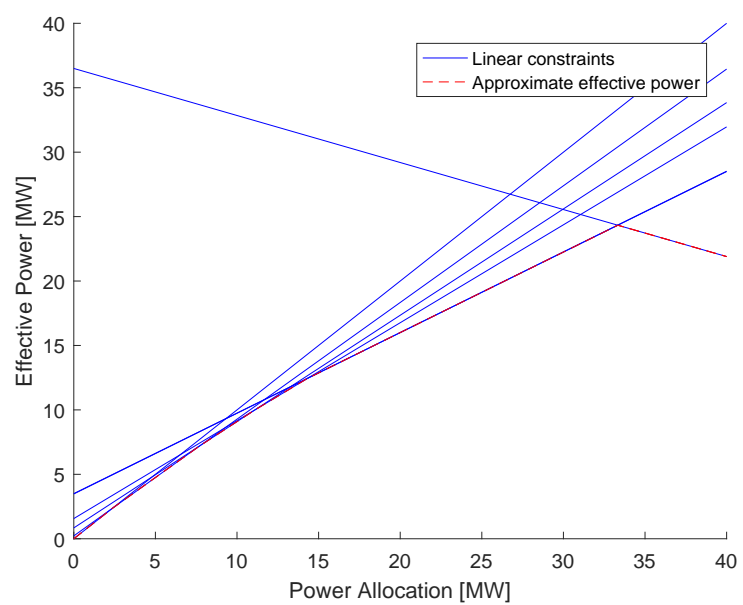

Figure 2. Effective power as a function of power allocation to frequency regulation for an energy allocation of $10 \mathrm{MWh}$.

Table 1. Coefficients used to compute $C^{\mathrm{FR}}$ and $P^{\mathrm{eFR}}$

\begin{tabular}{ccccc}
\hline$l$ & $a^{\mathrm{FR}, 1}$ & $b^{\mathrm{FR}, 1}$ & $a^{\mathrm{FR}, 2}$ & $b^{\mathrm{FR}, 2}$ \\
\hline 1 & -121.40 & 214.01 & -0.3649 & 3.6496 \\
2 & -22.32 & 15.86 & 0.6248 & 0.3503 \\
3 & -12.42 & 1.71 & 0.6260 & 0.3478 \\
4 & -12.24 & 1.52 & 0.7598 & 0.1566 \\
5 & -11.66 & 0.99 & 0.8251 & 0.0841 \\
6 & -11.08 & 0.65 & 0.9056 & 0.0222 \\
7 & -10.40 & 0.29 & 1.0 & 0.0000 \\
8 & -10.27 & 0.25 & - & - \\
9 & -10.19 & 0.22 & - & - \\
10 & -10.09 & 0.198 & - & - \\
11 & -10.06 & 0.191 & - & - \\
12 & 0.00 & 0.00 & - & - \\
\hline
\end{tabular}

and the optimization problem is separable, i.e., each battery can optimize its own allocations and will achieve the same solution as the aggregator would achieve if it co-optimized all battery allocations. However, if frequency regulation is contracted for longer durations than $k$ then the problem is no longer separable and, for all $t$,

$$
B_{t}^{\mathrm{FR}}=\sum_{i \in \mathcal{N}} P_{i, k}^{\mathrm{eFR}} \quad \forall k \in[\alpha, \beta]
$$

where $\alpha$ corresponds to the first time step $k$ in the market interval $t$ and $\beta$ corresponds to the last.

2.3.4. Demand Charge. We model the demand charge as

$$
C_{i}^{\mathrm{d}}=g^{\mathrm{d}} \bar{p}_{i}^{\mathrm{g}} \quad \forall i
$$


where $\bar{p}_{i}^{\mathrm{g}}=\max \left(-p_{i, 1}^{\mathrm{g}}, \ldots,-p_{i, K}^{\mathrm{g}}\right)$, which we model with

$$
\bar{p}_{i}^{\mathrm{g}} \geq-p_{i, k}^{\mathrm{g}} \quad \forall i, k
$$

\subsection{BES Losses}

In order to assess losses, we use the decision variables obtained from solving the optimization problem to compute the real-time power/energy trajectories. The power trajectory is

$$
p_{i, j}^{\mathrm{bat}}=p_{i, j}^{\mathrm{d}}-p_{i, j}^{\mathrm{c}}+\omega_{j}^{\mathrm{FR}} P_{i, k}^{\mathrm{eFR}}-p_{i, j}^{\mathrm{off}}
$$

where $\omega_{j}^{\mathrm{FR}}$ is the frequency regulation signal, $p_{i, j}^{\text {off }}$ is the offset power, and $p_{i, j}^{\mathrm{d}}, p_{i, j}^{\mathrm{c}}$ are the charging/discharging decisions, which are constant for all $j$ within a time step $k$. We use a PJM frequency regulation signal for $\omega^{\mathrm{FR}}$. Let $p_{i, j}^{\text {bat,c }}=-p_{i, j}^{\text {bat }}$ if $p_{i, j}^{\text {bat }}<0$ and $p_{i, j}^{\text {bat,c }}=0$ otherwise, and let $p_{i, j}^{\text {bat,d }}=p_{i, j}^{\text {bat }}$ if $p_{i, j}^{\text {bat }} \geq 0$ and $p_{i, j}^{\text {bat,d }}=0$ otherwise. Then, the energy state trajectory is

$$
E_{i, j+1}^{\mathrm{bat}}=E_{i, j}^{\mathrm{bat}}+\eta_{i}^{\mathrm{c}} p_{i, j}^{\mathrm{bat}, \mathrm{c}} \Delta j+\frac{p_{i, j}^{\mathrm{bat}, \mathrm{d}}}{\eta_{i}^{\mathrm{d}}} \Delta j
$$

where $E_{i, j}^{\text {bat }}$ is the charged energy level of the battery at time $j$. The offset power is recomputed every 5 minutes using

$$
p_{i, j}^{\mathrm{off}}=\left(E_{i, j}^{\mathrm{bat}}-\left(E_{i, j}^{\mathrm{l}}+S o C_{\mathrm{avg}}^{\mathrm{FR}} E_{i, j}^{\mathrm{FR}}\right)\right) / 5 \text { min }
$$

and then held constant until it is recomputed. The energy states $E_{i, j}^{1}$ are computed by linearly interpolating $E_{i, k}^{1}$. Offsetting every 5 minutes is consistent with California's Regulation Energy Management functionality which uses the real-time $(5 \mathrm{~min})$ market to manage the state of charge of nongenerating resources.

Finally, the total losses over the day are calculated as

$$
E_{i}^{\mathrm{loss}}=\sum_{j}\left(\left(1-\eta_{i}^{\mathrm{c}}\right) p_{i, j}^{\mathrm{bat}, \mathrm{c}} \Delta j+\left(1-\frac{1}{\eta_{i}^{\mathrm{d}}}\right) p_{i, j}^{\mathrm{bat}, \mathrm{d}} \Delta j\right)
$$

\section{Case Studies}

We solve the optimization problem using $\Delta k=1$ hour, $K=24$ hours, and a 2 hour frequency regulation contract duration for the set of 17 heterogeneous batteries (5 Solar, $2 \mathrm{C} / \mathrm{I}$, and 10 Residential) described in Table 2, using Gurobi 7.0.2 as our solver. We run three separate case studies.

Case Study 1 gives illustrative results. Residential hourly TOU rates are set to PG\&E residential rates
Table 2. Battery Parameters used in Case Studies

\begin{tabular}{lccc}
\hline Type & $\begin{array}{c}\text { Service } 1 \\
\text { Solar }\end{array}$ & $\begin{array}{c}\text { Service 2 } \\
\text { C/I }\end{array}$ & $\begin{array}{c}\text { Service 3 } \\
\text { Residential }\end{array}$ \\
\hline Index $(i)$ & $1-5$ & $6-7$ & $8-17$ \\
$E^{\text {cap }}[\mathrm{kWh}]$ & 200 & 200 & 14 \\
$P^{\text {cap }}[\mathrm{kW}]$ & 100 & 100 & 7 \\
$\eta^{\mathrm{c}}$ & 0.90 & 0.90 & 0.90 \\
$\eta^{\mathrm{d}}$ & 0.90 & 0.90 & 0.90 \\
$\operatorname{Max} G_{i}[\mathrm{~kW}]$ & 500 & - & - \\
$\operatorname{Max} L_{i}[\mathrm{~kW}]$ & - & 200 & 4 \\
$p^{\text {g,max }}$ & 450 & - & - \\
$S o C^{\min }$ & 0.15 & 0.15 & 0.15 \\
$S o C^{\max }$ & 0.85 & 0.85 & 0.85 \\
$S o C_{\text {avg }}^{\text {FR }}$ & 0.525 & 0.525 & 0.525 \\
\hline
\end{tabular}

[16]. TOU rates and demand charges for $\mathrm{C} / \mathrm{I}$ customers are from [17], which is for customers with demands of 200 - $499 \mathrm{~kW}$. Summer rates, secondary voltage, were used. The summer energy rate from [17] for medium-demand metered service, primary voltage was used as the constant for the PV purchase price. We use solar data from [18]. The data are minutely but we use the average over each hour and also scale it to a maximum of $500 \mathrm{~kW}$ over the day. We use two C/I load profiles obtained from PG\&E. The profiles correspond to that of a water utility and a bakery. The data are 15 minutely but we use the average over each hour and scale them to have a peak demand of $200 \mathrm{~kW}$. For the residential load profile, we selected a representative high load day in Ann Arbor, Michigan on July 1 from [19]. The maximum demand in an hour is approximately $4 \mathrm{~kW}$. Conservatively, we model all 10 residential profiles to be the same. Diversity of loads would allow more potential for aggregation. The frequency regulation revenue was set to $\$ 0.05$ per $\mathrm{kWh}$. Since we aim to investigate the impacts of providing frequency regulation on top of the local services, this value was selected to be lower than the economic benefits of the local services.

Case Study 2 explores the environmental impacts of multitasking. We compute the losses incurred by multitasking batteries providing each type of local service and compare them to the losses incurred by single-use batteries providing the same services. Specifically, we simulate an additional battery, sized to enable equivalent frequency regulation participation over the day ( $213 \mathrm{~kW}, 213 \mathrm{kWh})$, and compute its losses. We also investigate the required capacity of multitasking versus single-use batteries.

Case Study 3 is a sensitivity study on the power/energy capacity allocation results as a function of the prices. We assume the frequency regulation market 


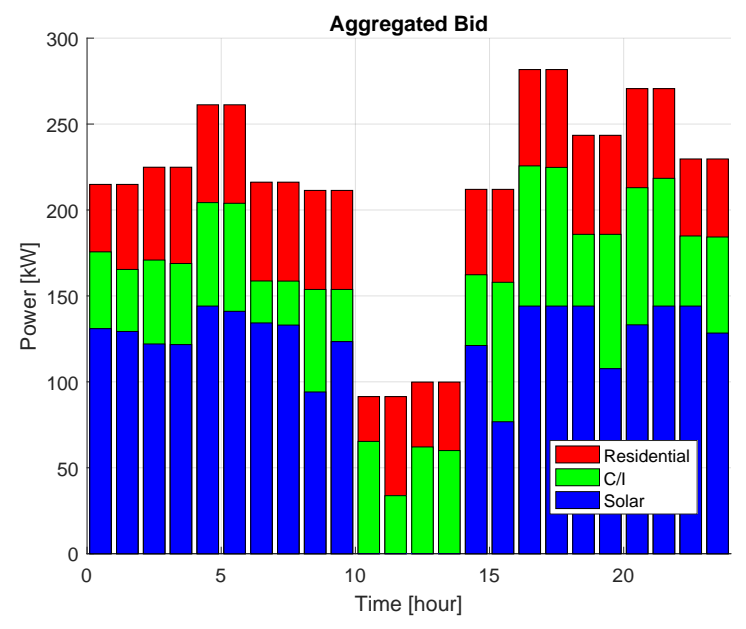

Figure 3. Aggregated bid to frequency regulation.

Table 3. Percent capacity bid to frequency regulation (FR)

\begin{tabular}{lccc}
\hline & $\begin{array}{c}\text { Average } \\
\text { FR bid } \\
{[\mathrm{kW}]}\end{array}$ & $\begin{array}{c}\text { Power } \\
\text { Capacity } \\
{[\mathrm{kW}]}\end{array}$ & $\begin{array}{c}\text { Percent } \\
\text { Capacity } \\
\text { Bid }\end{array}$ \\
\hline Service 1 & 106.7 & 175 & $61.0 \%$ \\
Service 2 & 54.8 & 100 & $54.8 \%$ \\
Service 3 & 47.5 & 70 & $68.0 \%$ \\
\hline
\end{tabular}

intervals $t$ are equivalent to the time steps $k$ ( 1 hour) and so we are able to optimize each battery individually.

\section{Results}

\subsection{Case Study 1: Illustrative Results}

The aggregated bid to frequency regulation and the portions provided by batteries providing each local service are shown in Fig. 3. The average frequency regulation capacity bid by batteries providing each local service and percent of capacity to frequency regulation are shown in Table 3. The power and energy allocations for each hour for each three of the batteries, each providing a different local service, are shown in Fig. 4.

For the aggregated BES capacity of $345 \mathrm{~kW}$, an hourly average of $213 \mathrm{~kW}$ was able to be bid into the frequency regulation market. Batteries providing Services 2 and 3 were able to allocate power to frequency regulation every hour. In contrast, batteries providing Service 1 were not because the solar inverter restricts BES from participating in frequency during solar PV's peak production hours when the full inverter capacity is used to feed solar PV electricity on to the grid.

Figure 5 shows the impact of BES on power flow
Table 4. Single-service BES Ratings and daily losses

\begin{tabular}{lccc}
\hline & $P^{\text {cap }}$ & $\begin{array}{c}\text { Number of } \\
{[\mathrm{kW}]}\end{array}$ & $\begin{array}{c}\text { Losses } \\
{[\mathrm{kWh}]}\end{array}$ \\
\hline Service 1 & 35 & 5 & $18.7 / \mathrm{BES}$ \\
Service 2 & 50 & 2 & $29.9 / \mathrm{BES}$ \\
Service 3 & 7 & 10 & $1.5 / \mathrm{BES}$ \\
FR Only & 213 & 1 & $316.0 / \mathrm{BES}$ \\
Total & 558 & 18 & 483.6 \\
\hline
\end{tabular}

Table 5. Multitasking BES Ratings and daily losses

\begin{tabular}{lccc}
\hline & $\begin{array}{c}P^{\text {cap }} \\
{[\mathrm{kW}]}\end{array}$ & $\begin{array}{c}\text { Number of } \\
\text { BES }\end{array}$ & $\begin{array}{c}\text { Losses } \\
{[\mathrm{kWh}]}\end{array}$ \\
\hline Service 1 & 35 & 5 & $45.7 / \mathrm{BES}$ \\
Service 2 & 50 & 2 & $57.2 / \mathrm{BES}$ \\
Service 3 & 7 & 10 & $8.1 / \mathrm{BES}$ \\
Total & 345 & 17 & 424.4 \\
\hline
\end{tabular}

to/from the grid for batteries providing each local service. We see a reduction in solar clipping, a reduction in $\mathrm{C} / \mathrm{I}$ peak load, and residential load shifting to lower cost hours.

One example real-time SoC trajectory is shown in Fig. 6. As shown, the offset power ensures the SoC trajectories stays within their SoC limits.

\subsection{Case Study 2: Environmental Impacts}

The daily energy losses for batteries providing each type of service and the total BES losses are shown in Tables 4 and 5. In this example, the total BES losses decrease when the batteries multitask. Specifically, we observe a $12 \%$ reduction in system losses. This also decreases offsetting needs. The amount of offset power required to maintain the SoC of the battery providing frequency regulation only over 24 hours was $311 \mathrm{kWh}$ while the total amount of offset power required for the multitasking batteries was $261 \mathrm{kWh}$. This $16.0 \%$ reduction in purchased energy would reduce system-wide emissions associated with the generation of the offset power.

As shown in Tables 4 and 5 multitasking also reduces the required installed capacity. A system of batteries providing single services equivalent to those provided by the system of multitasking batteries would need to have a total installed capacity of $558 \mathrm{~kW}$ as compared to $345 \mathrm{~kW}$, which is a $38 \%$ reduction. A reduction in installed capacity reduces the environmental burdens associated with the materials and manufacturing of the BES. 

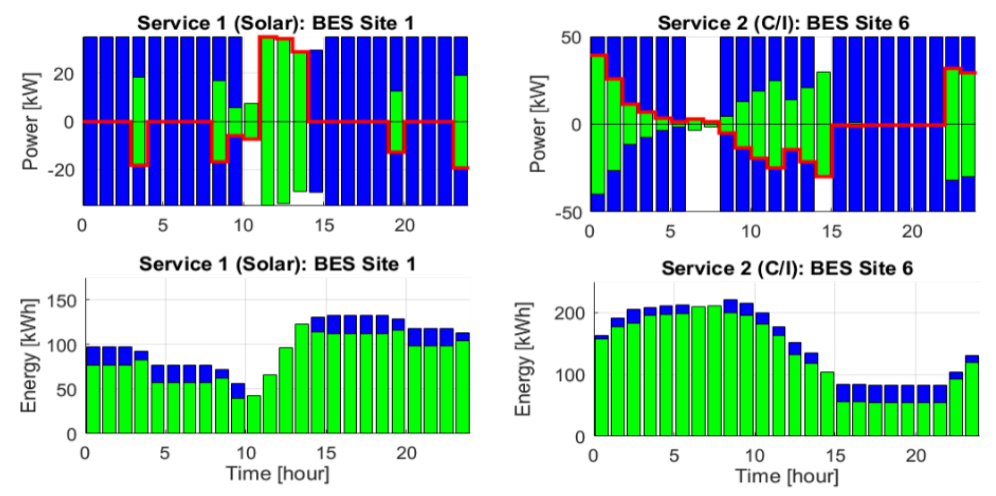
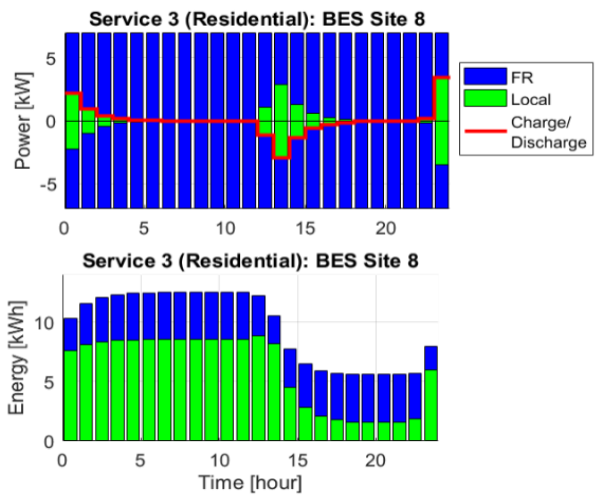

Figure 4. Example power and energy allocations.
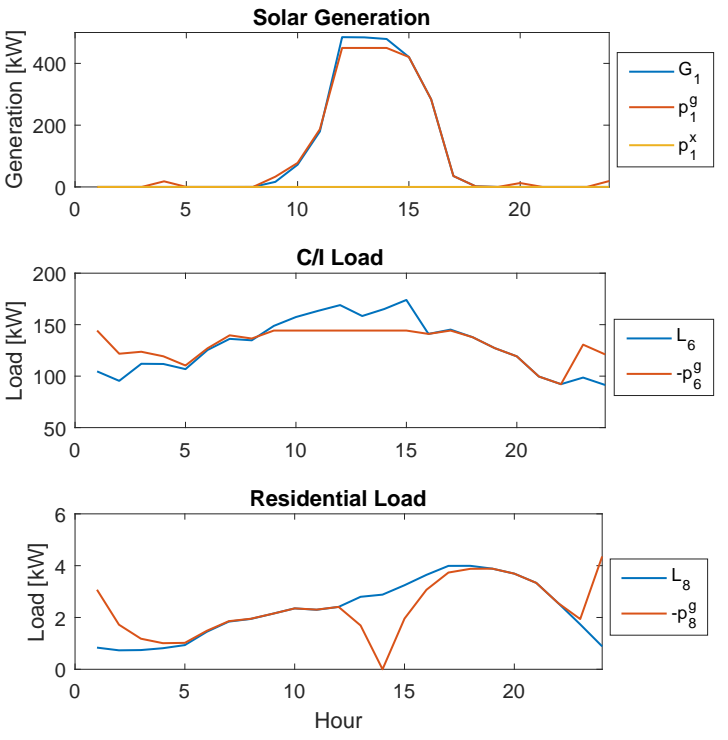

Figure 5. Example generation/load profiles (blue) and power flow to/from the grid with BES (red).

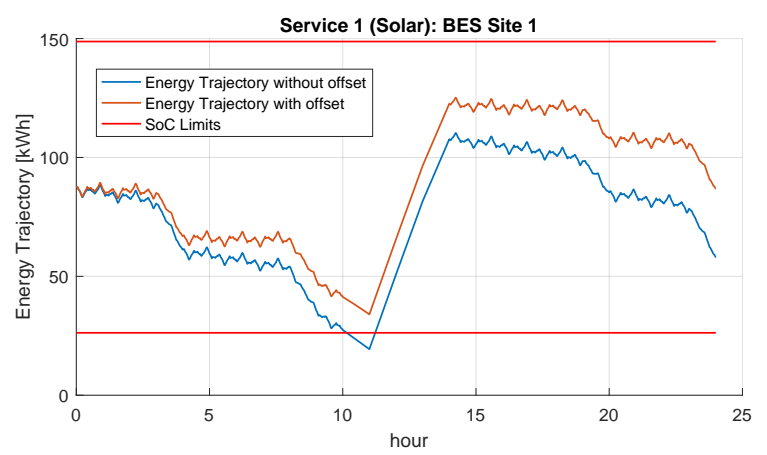

Figure 6. Example SoC Trajectory BES 1. 


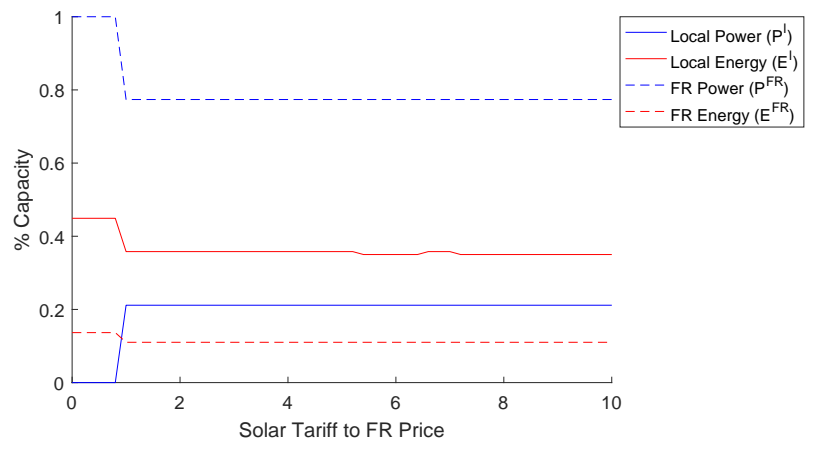

Figure 7. Power and energy allocations of a battery providing Service 1 as a function of the ratio between the solar tariff and frequency regulation (FR) price

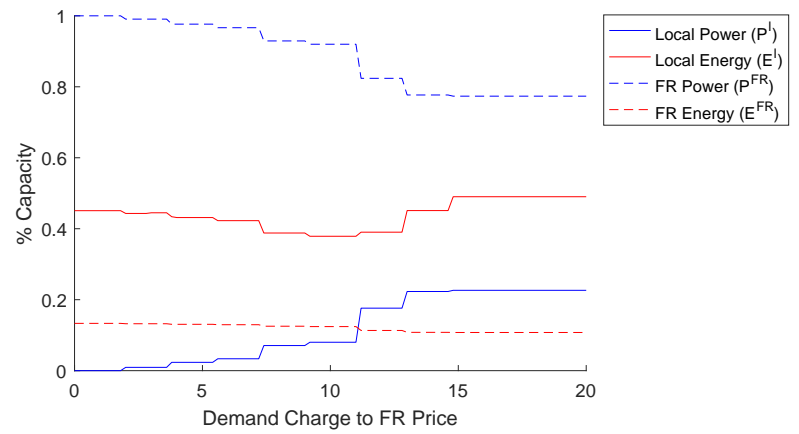

Figure 8. Power and energy allocations of a battery providing Service 2 as a function of the ratio between the demand charge and the frequency regulation (FR) price

local service under increasing ratios of TOU factor to frequency regulation price.

In Figs. 7 7 9 , we demonstrate the importance of relative prices in determining the optimal allocation of BES capacity to local and grid services. Prices vary greatly across different regions and energy markets, suggesting that optimal allocations of BES capacity and associated environmental impacts would likely vary significantly as well. Determining the precise environmental impact of BES multitasking in a particular system requires running analyses such as those conducted here but using parameters specific to the system of interest.

\section{Conclusions}

In this paper, we co-optimized multiple BES systems performing three different local services to enable provision of frequency regulation using the unused capacity of each system. We investigated the environmental impacts of multitasking versus

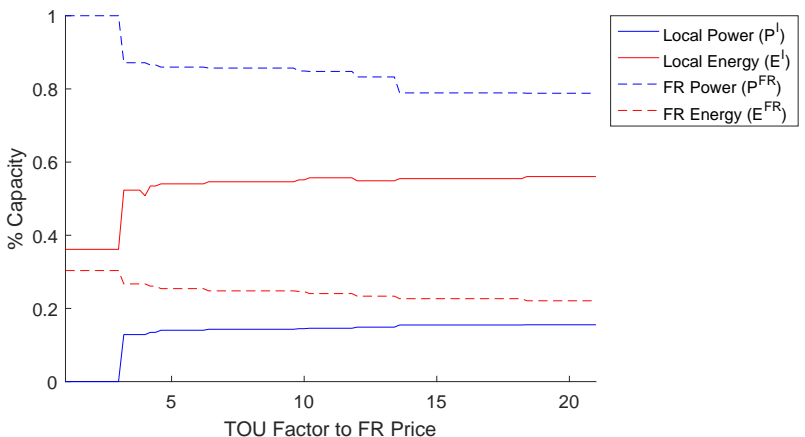

Figure 9. Power and energy allocations of a battery providing Service 3 as a function of the ratio between the TOU price factor and the frequency regulation (FR) price

single-tasking and found that aggregated multitasking can reduce the amount of installed BES capacity required to perform the same amount of services with individual batteries. We also observe reductions in BES energy losses. Both of these findings indicate that BES multitasking may result in reduced environmental impacts as compared to using BES systems only for single tasks.

Future work will determine how multitasking impacts BES degradation. Previous research has developed methods to control batteries while managing degradation [20, 21, 22]. Research is needed to determine how use of such control methods affects the environmental impacts of multitasking batteries.

\section{References}

[1] D. Kottick, M. Blau, and D. Edelstein, "Battery energy storage for frequency regulation in an island power system," IEEE Transactions on Energy Conversion, vol. 8, no. 3, pp. 455-459, 1993.

[2] R. Sioshansi, S. H. Madaeni, and P. Denholm, "A dynamic programming approach to estimate the capacity value of energy storage," IEEE Transactions on Power Systems, vol. 29, no. 1, pp. 395-403, 2014.

[3] A. Oudalov, R. Cherkaoui, and A. Beguin, "Sizing and optimal operation of battery energy storage system for peak shaving application," in IEEE PowerTech, pp. 621-625, IEEE, 2007.

[4] J. Eyer and G. Corey, "Energy storage for the electricity, grid: Benefits and market potential assessment guide," Sandia National Laboratories, vol. 20, no. 10, p. 5, 2010.

[5] X. Xi, R. Sioshansi, and V. Marano, "A stochastic dynamic programming model for co-optimization of distributed energy storage," Energy Systems, vol. 5, no. 3, pp. 475-505, 2014.

[6] S. Han, S. Han, and K. Sezaki, "Development of an optimal vehicle-to-grid aggregator for frequency regulation," IEEE Transactions on Smart Grid, vol. 1, no. 1 , pp. $65-72,2010$. 
[7] O. Mégel, J. L. Mathieu, and G. Andersson, "Scheduling distributed energy storage units to provide multiple services under forecast error," International Journal of Electrical Power \& Energy Systems, vol. 72, pp. 48-57, 2015.

[8] R. Moreno, R. Moreira, and G. Strbac, "A MILP model for optimising multi-service portfolios of distributed energy storage," Applied Energy, vol. 137, pp. 554-566, 2015.

[9] D. Wu, C. Jin, P. Balducci, and M. Kintner-Meyer, "An energy storage assessment: Using optimal control strategies to capture multiple services," in IEEE Power \& Energy Society General Meeting, 2015.

[10] O. Mégel, J. L. Mathieu, and G. Andersson, "Stochastic dual dynamic programming to schedule energy storage units providing multiple services," in IEEE PowerTech, 2015.

[11] E. Namor, F. Sossan, R. Cherkaoui, and M. Paolone, "Control of battery storage systems for the simultaneous provision of multiple services," IEEE Transactions on Smart Grid, 2018.

[12] Y. Shi, B. Xu, D. Wang, and B. Zhang, "Using battery storage for peak shaving and frequency regulation: Joint optimization for superlinear gains," IEEE Transactions on Power Systems, vol. 33, no. 3, pp. 2882-2894, 2018.

[13] G. Notton, V. Lazarov, and L. Stoyanov, "Optimal sizing of a grid-connected PV system for various pv module technologies and inclinations, inverter efficiency characteristics and locations," Renewable Energy, vol. 35, no. 2, pp. 541-554, 2010.

[14] M. Bolinger, J. Seel, and K. H. LaCommare, "Utility-scale solar 2016: An empirical analysis of project cost, performance, and pricing trends in the united states," tech. rep., Lawrence Berkeley National Lab, 2017.
[15] O. Mégel, J. L. Mathieu, and G. Andersson, "Maximizing the potential of energy storage to provide fast frequency control," in IEEE PES Innovative Smart Grid Technologies Europe, 2013.

[16] "Electric Schedule EM-TOU Residential Time of Use Service." https://www.pge.com/tariffs/ assets/pdf/tariffbook/ELEC_SCHEDS_EMTOU.pdf. Accessed: June 2018.

[17] "Electric Schedule A-10 Medium General Demand-Metered Service." https://www.pge. com/tariffs/assets/pdf/tariffbook/ ELEC_SCHEDS_A-10.pdf Accessed: June 2018.

[18] C. Maxey and A. Andreas, "Rotating shadowband radiometer (rsr) oak ridge, tennessee (data) NREL Report No. DA-5500-56512.." http://dx.doi. org/10.5439/1052553 2007.

[19] OpenEI, "Commercial and residential hourly load profiles for all tmy 3 locations in the united states." https://openei.org/datasets/dataset/ commercial-and-residential-hourlyload-profiles-for-all-tmy3-locationsin-the-united-states Accessed: June 2018.

[20] M. Koller, T. Borsche, A. Ulbig, and G. Andersson, "Defining a degradation cost function for optimal control of a battery energy storage system," in IEEE PowerTech, 2013.

[21] A. Perez, R. Moreno, R. Moreira, M. Orchard, and G. Strbac, "Effect of battery degradation on multi-service portfolios of energy storage," IEEE Transactions on Sustainable Energy, vol. 7, no. 4, pp. 1718-1729, 2016.

[22] J. L. Mathieu and J. A. Taylor, "Controlling nonlinear batteries for power systems: Trading off performance and battery life," in Power Systems Computation Conference, 2016. 